\title{
Studies on Morpho-Physiological Traits Associated with Drought Tolerance in Local Landraces of Rice (Oryza sativa L.)
}

\author{
G.T. Navya*, B.M. Dushyanthakumar, R. Madhuri, K.N. Shubha and S. Gangaprasad
}

Department of Genetics and Plant Breeding, College of Agriculture, University of Agricultural and Horticultural Sciences, Navile, Shimoga, India

*Corresponding author

\section{A B S T R A C T}

Productivity of rice is negatively affected by drought. Morpho-physiological traits are key for plant adaptation under water stress condition. This study was conducted with

\section{Keywords}

Drought stress,

Grain yield,

Physiological traits,

Relative water

content, Root

length, Proline

content

Article Info

Accepted:

15 June 2019

Available Online:

10 July 2019 the objective to study the 49 local land races of rice including checks in field and PVC pipes for screening drought tolerant genotypes based on their yield performance and the effect of various physiological traits associated with drought tolerance were studied. Results revealed significant yield decline in almost all the genotypes studied under drought condition as compared to irrigated condition. Yield per plant was significantly and positively associated with productive tillers per plant, number of spikelets per panicle, number of filled grains per panicle, seed filling per cent and test weight. Physiological characters like chlorophyll content, relative water content, proline content, root length, root to shoot ratio and root volume showed significant positive correlation with yield under drought condition. Phenotypic path-coefficient analysis revealed that test weight in drought and number of spikelets per panicle under control showed highest direct effect on yield per plant and among physiological characters root length under drought and control condition exhibited highest direct effect on yield. However, out of 49 genotypes Sannavaalya, Manjakaime, JGL-1798, Gangadace, Madras sanna and Najarbad showed superiority in terms of grain yield and its associated traits.

\section{Introduction}

Rice (Oryza sativa L.) is the primary staple food crop for about 65 per cent of the world population and influences the livelihoods and economies of several billion people. It is cultivated in all the continents of the world except Antarctica. Asia is the major producer and consumer of rice. In India the crop is cultivated in an area of 44.1 million hectares, with a production of 105.50 million tonnes and productivity of $2391.00 \mathrm{~kg} / \mathrm{ha}(2014-15)$. In 2016 the production of rice decreased to 104.4 million tonnes (Anonymous, 2016).

The frequent occurrences of abiotic stresses have been identified as the key to the low rice productivity of rainfed ecosystem. Among all the abiotic stresses, salinity, drought, submergence and mineral toxicity are the most 
important limiting factors affecting rice production. In Asia, rice cultivation generally follows a bimodal rainfall pattern, thus the drought stress during the growing season can be classified into three types, namely early stress (early in the growing season), intermittent stress (in the middle of the growing season) and late stress (in the late growing season) (Chang et al., 1979). About 23 million hectares of rainfed rice in Asia are estimated to be drought prone, and drought is becoming an increasing problem even in traditionally irrigated areas of India (Pandey et al., 2005).

There is a global consensus that current climate is changing mainly due to the anthropogenic emissions of greenhouse gases. There is widespread concern over long term climate changes and change in climate variability through occurrence of extreme weather events such as cyclone, floods, drought and sea level raise etc. The impact of climate change on cropping pattern in dry land regions have emerged as a critical concern in recent years (Naveen et al., 2011). Drought is "a shortfall of water availability sufficient to cause loss in yield" or a period of no rainfall or irrigation that affects crop growth. Yield of rainfed lowland rice, which occupies about 25 per cent of the world's rice areas, are drastically reducing by drought due to unpredictable, insufficient and uneven rainfall during the growing period (Zulqarnain et al., 2013). So, the Plants have evolved several adaptive mechanisms which classified into four categories to overcome the effect of stress. Three of these adaptations are developmental traits (e.g. time of flowering), structural traits (e.g. leaf waxiness) and physiological mechanisms (e.g. maintaining high relative water content) involve complex interaction. The fourth one is the metabolic responses such as alteration in metabolism and accumulation of organic osmolytes, most commonly proline (Jabasingh and Saravana.
2013). The root characteristics also play an important role in drought tolerance, depending on the genotypes, the roots have the ability to undergo developmental changes at different extents to better adapt to water stress.

Hence, it is essential to study the degree of association between these mechanisms with yield attributing characters. A study of correlation and path analysis thus help to identify suitable selection criteria for improving yield. In this context present study was conducted to study genetic variability and association between morpho-physiological traits with yield under drought stress condition.

\section{Materials and Methods}

\section{Experimental material and site}

The present study was conducted during summer 2016 at College of Agriculture, University of Agricultural and Horticultural Sciences, Shivamogga in the field experiment plots of department of Genetics and Plant Breeding. The experimental material consisting of 45 local land races of rice and three checks Anagha (resistant check), Jyothi (susceptible check), JGL-1798 and Poustic 9.

The experiment was laid out in Randomized Complete Block Design (RCBD) with two replications under control and drought stress condition.

The normal package of practices was followed except for irrigation schedule, Moisture stress was imposed 30 days after transplanting by withholding irrigation and only lifesaving flash irrigation was given when plants showed wilting symptoms, whereas in control condition regular irrigation was given without creation of any stress. The spacing maintained was $20 \mathrm{~cm}$ between rows and $10 \mathrm{~cm}$ between plants within a row. 


\section{Sampling and observation}

Observation on yield and yield attributing traits were recorded on five randomly selected plants per genotype for days to 50 per cent flowering (DFF), days to maturity (DM), number of total tillers per plant (TT), number of productive tillers per plant (PT), number of spikelets per panicle (SPP), number of filled grains per panicle (FGPP), panicle length (PL), plant height (PH), test weight (TW) and seed filling per cent (SFP).

\section{Studies on physiological parameters}

Root traits were studied in PVC pipes of 100 $\mathrm{cm}$ length and $20 \mathrm{~cm}$ diameter was used. The pipes were filled with a mixture of red soil and FYM. Seeds were directly sown in the pipes. After 30 days irrigation was withheld to create water stress whereas, for control regular irrigation was given without creating stress. At the time of flowering, the pipes were carefully removed and immersed in water for few hours to loosen the soil then washed with a jet of water. Then the plants were carefully removed and washed with water without damaging the roots then observations like root length (RL), shoot length (SL), root volume (RV) were recorded.

Chlorophyll content was measured using SPAD chlorophyll meter (KONICA MINOLTA, SPAD 502 PLUS, Version: 1.20.0000) in random five leaves of each genotype at flowering stage.

Leaf relative water content (RWC) was estimated by recording fresh weight then leaf sample was immersed in water for an overnight and turgid weight was recorded then samples were kept in hot air oven and recorded dry weighttill constant weight was achieved and relative water content was calculated using the formula given by (Weatherley. 1950)
Relative water content $(\%)=\frac{\text { Fresh weight-Dry weight }}{\text { Turgid weight-Dry weight }} \times 100$

Proline content was estimated as per Bates et al., (1973).0.5 g of leaf sample was collected and homogenized with $10 \mathrm{ml} 3$ percent aqueous Sulpho-salicilic acid in pestle and morter then filtered. Filtrate volume was made up to $25 \mathrm{ml}$ then $2 \mathrm{ml}$ was pipetted out into test tubes. $2 \mathrm{ml}$ of filtrate was reacted with $2 \mathrm{ml}$ acid ninhydrin and $2 \mathrm{ml}$ of glacial acetic acid for one hour at $100^{\circ} \mathrm{C}$, and the reaction terminated in an ice bath. The reaction mixture was extracted with $4 \mathrm{ml}$ toluene, mixed vigorously with a test tube stirrer for 15-20 sec. the chromophore containing tolune was aspirated from the aqueous phase, warmed to room temperature and the absorbance read at $520 \mathrm{~nm}$ in a spectrophotometer. The proline concentration was determined from a standard curve and calculated on a fresh weight basis.

Proline $(\mu \mathrm{mole} / \mathrm{g} F W)=\frac{(\mu \mathrm{g} \text { proline } / \mathrm{ml} \mathrm{X} \mathrm{ml} \mathrm{toluene}) / 115.5 \mathrm{\mu g} / \mathrm{mole}}{\text { g sample }}$

\section{Results and Discussion}

The results related to performance of yield and yield attributing traits of selected rice genotypes under control and drought condition have been presented in Table 1. Crop under drought condition produced significantly lower yield per plant when compared with the irrigated control condition. The range of yield reduction ranged from $38.39 \%$ to $90 \%$. In general, across the genotypes 2 to 28 days early flowering was observed under drought condition when compared with irrigated condition. However, the responses varied among genotype. Santhosh et al., (2014) reported 29 to $78 \%$ yield reduction under drought condition and observed nonsignificant delay in fifty per cent flowering under water stress condition as compared to non-stress irrigated situation. Reduced number of productive tillers per plant, seed filling 
percent, yield per plant, seed filling per cent was observed under drought condition when compared to control condition. Similar trends were also observed for physiological parameters chlorophyll content and relative water content whereas, increased proline content, root length and root volume under drought condition. Maisura et al., (2014) reported decrease in chlorophyll content and increased proline accumulation under drought stress. Ganapathy et al., (2010) reported significant superior mean values than grand mean for root traits in drought tolerant lines.

The analysis of variance was significant for all the yield attributing morphological characters under both drought and control conditions are present in Table 2 and 3 respectively. Similar results were reported by Uday (2013).

\section{Correlation and path analysis between yield and its attributes}

Primary aim of any breeding program is to increase yield. The yield of the crop depends on the performance of its matrix trait known as yield attributing traits. Inter-relationship between grain yield and yield attributes were determined by correlation matrix studied presented in Table 4. The yield per plant was significantly and positively associated with productive tillers per plant, number of spikelets per panicle, number of filled grains per panicle, seed filling per cent and test weight under drought stress condition whereas, under control condition yield was positive association with days to 50 per cent flowering and seed filling per cent. Santosh et al., (2014) reported positive and significant correlation for yield with harvest index and tiller number under both drought stress and non-stress irrigated condition. Manickavelu et al., (2006) reported significant and positive correlation of yield with tillers per plant, grains per panicle, test weight under both stress and control condition whereas, days to flowering, plant height, panicle length under stress condition showed positive significant correlation with yield.

Correlation coefficient gives an idea of association between characters. But a dependant character is an interaction product of many mutually associated component characters and change system is studied using path coefficient analysis presented in Table 5. In the present study days to 50 per cent flowering, number of tillers per plant, number of spikelets per panicle, seed filling per cent under both drought stress and control condition, days to maturity, number of productive tillers per plant, number of filled grains per panicle, and test weight under drought, whereas plant height under control condition exhibited positive direct effect on yield per plant at phenotypic level. The results were in accordance with Manickavelu et al., (2006).

The plants were scored for leaf rolling (LR), using 0-9 standard evaluation system of rice

(Gana. 2011)

\begin{tabular}{|c|l|}
\hline Scale & \multicolumn{1}{|c|}{ Description } \\
\hline $\mathbf{0}$ & Leaves healthy. \\
$\mathbf{1}$ & Leaves starts to fold. \\
$\mathbf{3}$ & Leaves folding (V-shaped) \\
$\mathbf{5}$ & Leaves fully cupped (U- shaped) \\
$\mathbf{7}$ & Leaves margins touching (O- shaped) \\
$\mathbf{9}$ & Leaves tightly rolled. \\
\hline
\end{tabular}


Table.1 Drought tolerant genotypes identified and their respective traits under drought and control condition

\begin{tabular}{|c|c|c|c|c|c|c|c|c|c|c|c|c|c|c|}
\hline \multirow[t]{2}{*}{ Genotype } & \multicolumn{2}{|c|}{ DFF } & \multicolumn{2}{|c|}{ YPP (g) } & \multicolumn{2}{|c|}{$\operatorname{SFP}(\%)$} & \multicolumn{2}{|c|}{$\begin{array}{l}\text { Chlorophyll } \\
\text { (spad) }\end{array}$} & \multicolumn{2}{|c|}{$\begin{array}{c}\text { Proline } \\
(\mu \mathrm{mole} / \mathrm{g} F W)\end{array}$} & \multicolumn{2}{|c|}{ RWC (\%) } & \multicolumn{2}{|c|}{$\begin{array}{l}\text { Root length } \\
\text { (cm) }\end{array}$} \\
\hline & D & C & D & C & D & C & D & C & D & C & D & C & D & C \\
\hline Sannavaalya & 79.00 & 90.00 & 24.75 & 35.12 & 90.25 & 91.85 & 15.20 & 16 & 18.56 & 14.15 & 78.28 & 83.70 & 36.00 & 17.00 \\
\hline Manjakaime & 72.50 & 84.00 & 22.85 & 37.09 & 89.23 & 95.01 & 14.00 & 15.80 & 17.25 & 12.56 & 72.30 & 86.25 & 35.00 & 21.00 \\
\hline Gangadace & 68.00 & 87.50 & 21.25 & 34.50 & 80.60 & 89.30 & 14.20 & 15.50 & 20.25 & 14.56 & 83.06 & 85.15 & 30.20 & 20.00 \\
\hline Madras sanna & 95.00 & 100.00 & 20.85 & 37.00 & 90.33 & 96.44 & 14.40 & 15.00 & 17.04 & 12.65 & 78.50 & 82.24 & 27.00 & 18.00 \\
\hline Najarbad & 83.50 & 91.50 & 20.25 & 33.85 & 80.62 & 90.29 & 16.12 & 17.45 & 19.35 & 14.15 & 76.21 & 79.52 & 28.00 & 18.60 \\
\hline JGL-1798 & 98.00 & 100.00 & 21.75 & 44.75 & 89.01 & 93.62 & 13.00 & 14.00 & 19.35 & 14.15 & 68.45 & 72.94 & 30.00 & 19.50 \\
\hline Anagha & 77.00 & 81.00 & 24.25 & 40.53 & 92.95 & 91.91 & 17.54 & 19.00 & 18.35 & 13.15 & 74.23 & 85.00 & 40.50 & 25.00 \\
\hline Jyoti & 80.50 & 90.00 & 8.75 & 34.25 & 46.45 & 92.91 & 6.90 & 11.00 & 21.25 & 12.15 & 52.15 & 84.56 & 18.20 & 15.50 \\
\hline Poustic 9 & 83.00 & 80.00 & 12.15 & 39.37 & 58.03 & 95.57 & 11.60 & 15.80 & 20.56 & 13.25 & 51.88 & 80.50 & 21.20 & 16.50 \\
\hline CD (5\%) & 5.75 & 2.68 & 2.58 & 6.28 & 9.64 & 7.70 & 3.26 & 3.15 & 3.11 & 2.07 & 7.38 & & 3.71 & \\
\hline CV (\%) & 3.61 & 1.40 & 18.28 & 8.14 & 11.73 & 4.00 & 15.37 & 10.36 & 6.57 & 7.23 & 10.12 & & 8.89 & \\
\hline
\end{tabular}

Table.2 Analysis of variance for yield and yield attributing characters in local land races of rice under drought conditions

\begin{tabular}{|c|c|c|c|c|c|c|c|c|c|c|c|c|}
\hline $\begin{array}{l}\text { Source of } \\
\text { variation }\end{array}$ & df & $\begin{array}{c}\text { Days to } \\
50 \% \\
\text { flowering }\end{array}$ & $\begin{array}{l}\text { Days to } \\
\text { maturity }\end{array}$ & $\begin{array}{l}\text { Total } \\
\text { tillers/plant }\end{array}$ & $\begin{array}{l}\text { Productive } \\
\text { tillers/plant }\end{array}$ & $\begin{array}{l}\text { Spikelets } \\
\text { / panicle }\end{array}$ & $\begin{array}{l}\text { Filled } \\
\text { grains / } \\
\text { panicle }\end{array}$ & $\begin{array}{l}\text { Panicle } \\
\text { length }\end{array}$ & $\begin{array}{l}\text { Plant } \\
\text { height }\end{array}$ & $\begin{array}{l}\text { Yield / } \\
\text { plant }\end{array}$ & $\begin{array}{l}\text { Seed } \\
\text { filling per } \\
\text { centage }\end{array}$ & $\begin{array}{l}\text { Test } \\
\text { weight }\end{array}$ \\
\hline Replication & 1 & 26.54 & 2.29 & 71.11 & 0.25 & 62.08 & 119.02 & 15.44 & 67.06 & 0.34 & 54.64 & 0.0009 \\
\hline Treatments & 48 & $205.65 * *$ & $205.89 * *$ & $33.45 * *$ & $29.26 * *$ & $1625.42 * *$ & $2539.74 * *$ & $7.53 * *$ & $226.76 * *$ & $92.73 * *$ & $1312.51 * *$ & $29.50 * *$ \\
\hline Error & 48 & 8.18 & 4.35 & 14.49 & 1.56 & 33.74 & 828.97 & 2.24 & 112.55 & 1.65 & 23.01 & 0.83 \\
\hline CD $(5 \%)$ & & 5.75 & 4.19 & 7.65 & 2.51 & 11.68 & 8.35 & 3.01 & 21.33 & 2.58 & 9.64 & 1.83 \\
\hline $\mathrm{CV}(\%)$ & & 3.61 & 1.88 & 22.00 & 10.84 & 7.18 & 11.41 & 7.88 & 12.42 & 18.28 & 11.73 & 5.65 \\
\hline
\end{tabular}


Table.3 Analysis of variance for yield and yield attributing characters in local land races of rice under control conditions

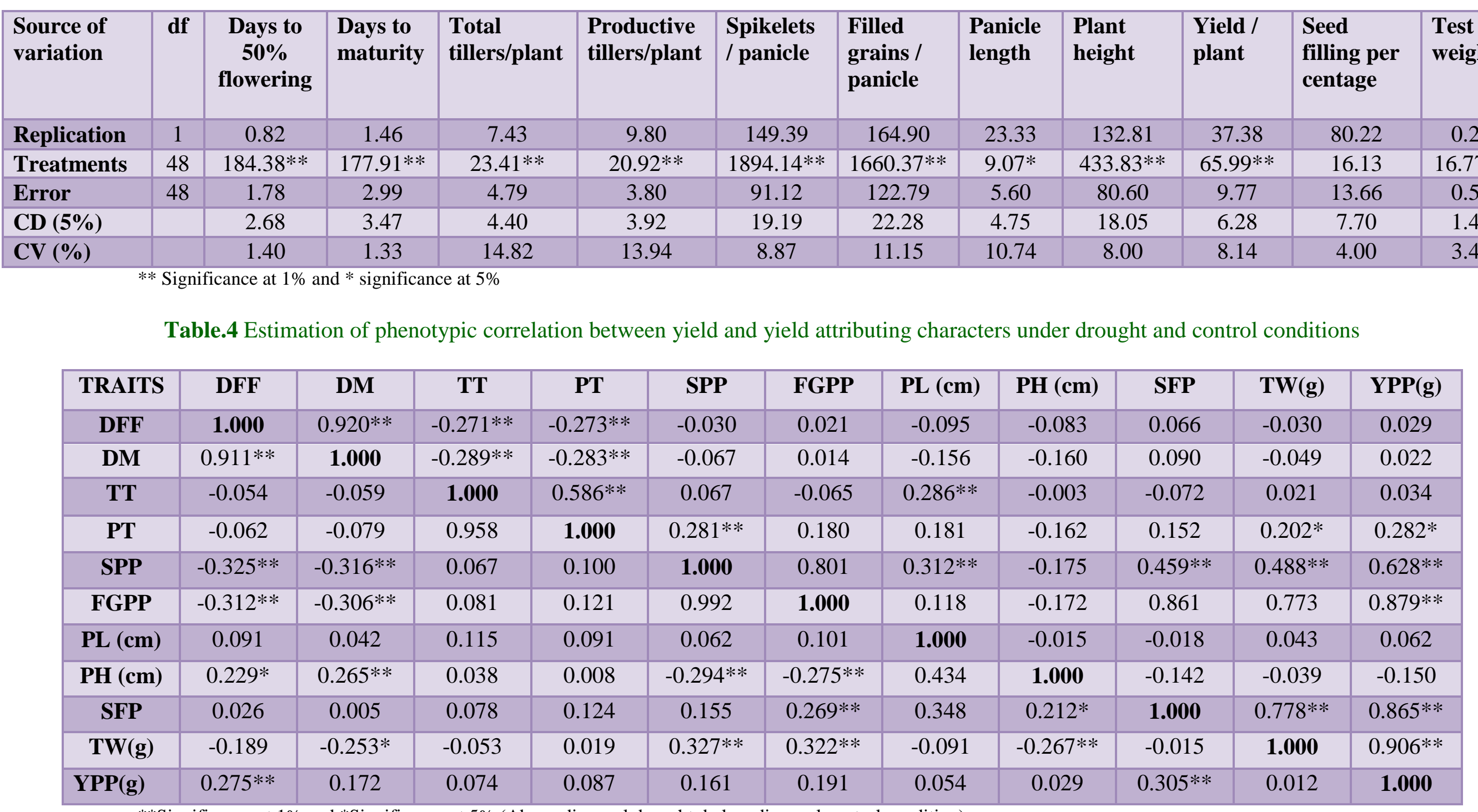

$* *$ Significance at $1 \%$ and $*$ Significance at $5 \%$.(Above diagonal drought; below diagonal control condition) 
Table.5a Estimates of direct and indirect effects of yield components on yield at phenotypic level under drought condition

\begin{tabular}{|c|c|c|c|c|c|c|c|c|c|c|}
\hline Traits & DFF & DM & TT & PT & SPP & FGPP & PL (cm) & PH $(\mathbf{c m})$ & SFP & TW $(\mathbf{g})$ \\
\hline DFF & $\mathbf{0 . 0 3 4 2}$ & 0.0315 & -0.0093 & -0.0093 & -0.0010 & 0.0007 & -0.0033 & -0.0028 & 0.0023 & -0.0011 \\
\hline DM & 0.0135 & $\mathbf{0 . 0 1 4 6}$ & -0.0042 & -0.0041 & -0.0010 & 0.0002 & -0.0023 & -0.0023 & 0.0013 & -0.0007 \\
\hline TT & -0.0038 & 0.0040 & $\mathbf{0 . 0 1 3 9}$ & 0.0082 & 0.0009 & -0.0009 & 0.0040 & -0.0001 & -0.0010 & 0.0003 \\
\hline PT & -0.0206 & -0.0214 & 0.0442 & $\mathbf{0 . 0 7 5 4}$ & 0.0212 & 0.0136 & 0.0137 & -0.0123 & 0.0115 & 0.0153 \\
\hline SPP & -0.0054 & -0.0121 & 0.0121 & 0.0505 & $\mathbf{0 . 1 7 9 5}$ & 0.1438 & 0.0560 & -0.0314 & 0.0824 & 0.0877 \\
\hline FGPP & 0.0006 & 0.0004 & -0.0019 & 0.0052 & 0.0230 & $\mathbf{0 . 0 2 8 7}$ & 0.0034 & -0.0049 & 0.0247 & 0.0222 \\
\hline PL (cm) & 0.0025 & 0.0041 & -0.0075 & -0.0048 & -0.0082 & -0.0031 & $-\mathbf{0 . 0 2 6 3}$ & 0.0004 & 0.0005 & -0.0011 \\
\hline PH (cm) & 0.0024 & 0.0047 & 0.0001 & 0.0047 & 0.0051 & 0.0050 & 0.0004 & $\mathbf{- 0 . 0 2 9 2}$ & 0.0041 & 0.0011 \\
\hline SFP & 0.0221 & 0.0301 & -0.0239 & 0.0506 & 0.1527 & 0.2865 & -0.0062 & -0.0473 & $\mathbf{0 . 3 3 2 5}$ & 0.2587 \\
\hline TW(g) & -0.0161 & -0.0260 & 0.0115 & 0.1061 & 0.2559 & 0.4048 & 0.0228 & -0.0206 & 0.4074 & $\mathbf{0 . 5 2 3 6}$ \\
\hline r value & $\mathbf{0 . 0 2 9 4}$ & $\mathbf{0 . 0 2 9 9}$ & $\mathbf{0 . 0 3 5}$ & $\mathbf{0 . 2 8 2 5}$ & $\mathbf{0 . 6 2 8 1}$ & $\mathbf{0 . 8 7 9 3}$ & $\mathbf{0 . 0 6 2 2}$ & $\mathbf{- 0 . 1 5 0 5}$ & $\mathbf{0 . 8 6 5 7}$ & $\mathbf{0 . 9 0 6}$ \\
\hline
\end{tabular}

Residual effect $=0.27$

Table.5b Estimates of direct and indirect effects of yield components on yield at phenotypic level under control condition

\begin{tabular}{|c|c|c|c|c|c|c|c|c|c|c|}
\hline Traits & DFF & DM & TT & PT & SPP & FGPP & PL (cm) & PH (cm) & SFP & TW(g) \\
\hline DFF & 0.7666 & 0.6985 & -0.0417 & -0.0475 & -0.2498 & -0.2398 & 0.0699 & 0.1755 & 0.0200 & -0.1453 \\
\hline DM & -0.4121 & -0.4523 & 0.0271 & 0.0359 & 0.1430 & 0.1385 & -0.0193 & -0.1200 & -0.0023 & 0.1147 \\
\hline TT & -0.0087 & -0.0096 & 0.1599 & 0.1532 & 0.0107 & 0.0130 & 0.0185 & 0.0062 & 0.0125 & -0.0086 \\
\hline PT & 0.0059 & 0.0075 & -0.0906 & -0.0946 & -0.0095 & -0.0115 & -0.0087 & -0.0008 & -0.0117 & -0.0018 \\
\hline SPP & -0.3773 & -0.3661 & 0.0777 & 0.1164 & 1.1580 & 1.1487 & 0.0728 & -0.3410 & 0.1796 & 0.3787 \\
\hline FGPP & 0.2943 & 0.2881 & -0.0764 & -0.1141 & -0.9332 & -0.9407 & -0.0956 & 0.2592 & -0.2534 & -0.3031 \\
\hline PL (cm) & -0.0120 & -0.0056 & -0.0153 & -0.0121 & -0.0083 & -0.0134 & -0.1321 & -0.0574 & -0.0460 & 0.0121 \\
\hline PH (cm) & 0.0035 & 0.0041 & 0.0006 & 0.0001 & -0.0046 & -0.0043 & 0.0067 & 0.0155 & 0.0033 & -0.0041 \\
\hline SFP & 0.0105 & 0.0021 & 0.0315 & 0.0501 & 0.0625 & 0.1085 & 0.1403 & 0.0857 & 0.4029 & -0.0063 \\
\hline TW(g) & 0.0045 & 0.0060 & 0.0013 & -0.0005 & -0.0078 & -0.0077 & 0.0022 & 0.0064 & 0.0004 & -0.0238 \\
\hline r value & 0.2752 & 0.1727 & 0.0741 & 0.0869 & 0.161 & 0.1913 & 0.0547 & 0.0293 & 0.3053 & 0.0125 \\
\hline
\end{tabular}

Residual effect $=0.68$ 
Table.6 Estimation of phenotypic correlation between yield and physiological characters under drought and control condition

\begin{tabular}{|c|c|c|c|c|c|c|c|c|c|}
\hline Traits & $\begin{array}{c}\text { Chlorophyll } \\
\text { SPAD } \\
\text { reading) }\end{array}$ & RWC (\%) & $\begin{array}{c}\text { Proline } \\
(\mu \mathrm{mole} / \mathrm{g} \text { FW) }\end{array}$ & LR & RL (cm) & SL (cm) & $\mathbf{R} / \mathbf{S}$ & $\mathbf{R V}(\mathrm{ml})$ & YPP(g) \\
\hline Chl & 1.000 & $0.544 * *$ & $0.558 * *$ & $-0.477 * *$ & $0.558 * *$ & -0.002 & $0.435 * *$ & $0.508 * *$ & $0.599 * *$ \\
\hline RWC & -0.044 & 1.000 & $0.679 * *$ & $-0.505 * *$ & $0.660 * *$ & 0.022 & $0.524 * *$ & $0.616 * *$ & $0.693 * *$ \\
\hline Proline & 0.063 & 0.069 & 1.000 & $-0.518 * *$ & $0.623 * *$ & 0.019 & $0.471 * *$ & $0.624 * *$ & $0.805 * *$ \\
\hline LR & $-0.232 *$ & 0.191 & $-0.379 * *$ & 1.000 & $-0.757 * *$ & 0.129 & $-0.653 * *$ & $-0.670 * *$ & $-0.687 * *$ \\
\hline RL & 0.026 & $0.239 *$ & $0.375^{*} *$ & -0.012 & 1.000 & -0.112 & $0.861 * *$ & $0.790 * *$ & $0.816 * *$ \\
\hline SL & 0.029 & 0.163 & 0.036 & $-0.242 *$ & -0.123 & 1.000 & $-0.572 * *$ & -0.071 & -0.080 \\
\hline $\mathbf{R} / \mathbf{S}$ & 0.039 & 0.074 & $0.250 *$ & 0.108 & $0.725 * *$ & $-0.712 * *$ & 1.000 & 0.665 & $0.679 * *$ \\
\hline $\mathbf{R V}$ & -0.032 & $0.279 * *$ & $0.370 * *$ & $-0.219 *$ & $0.347 * *$ & 0.089 & 0.177 & 1.000 & $0.777 * *$ \\
\hline YPP & 0.144 & -0.040 & -0.025 & -0.039 & 0.072 & -0.088 & 0.069 & 0.061 & 1.000 \\
\hline
\end{tabular}

**Significance at $1 \%, \quad *$ Significance at $5 \%$. (Above diagonal drought; below diagonal control condition)

Table.7a Estimates of direct and indirect effects of physiological parameters on yield at phenotypic level under drought condition

\begin{tabular}{|c|c|c|c|c|c|c|c|c|}
\hline Traits & Chl & RWC & Proline & LR & RL & SL & R/S & RV \\
\hline Chl & $\mathbf{0 . 0 5 0 8}$ & 0.0277 & 0.0284 & -0.0243 & 0.0284 & -0.0001 & 0.0222 & 0.0259 \\
\hline RWC & 0.0231 & $\mathbf{0 . 0 4 2 5}$ & 0.0288 & -0.0215 & 0.0280 & 0.0010 & 0.0223 & 0.0262 \\
\hline Proline & 0.2255 & 0.2743 & $\mathbf{0 . 4 0 3 7}$ & -0.2094 & 0.2515 & 0.0078 & 0.1901 & 0.2522 \\
\hline LR & 0.0330 & 0.0349 & 0.0358 & $\mathbf{- 0 . 0 6 9 1}$ & 0.0523 & -0.0089 & 0.0451 & 0.0463 \\
\hline RL & 0.2552 & 0.3017 & 0.2847 & -0.3462 & $\mathbf{0 . 4 5 7 0}$ & -0.0515 & 0.3936 & 0.3612 \\
\hline SL & 0.0003 & -0.0027 & -0.0023 & -0.0152 & 0.0133 & $\mathbf{- 0 . 1 1 7 7}$ & 0.0679 & 0.0084 \\
\hline R/S & -0.0775 & -0.0933 & -0.0838 & 0.1162 & -0.1532 & 0.1019 & $\mathbf{- 0 . 1 7 7 9}$ & -0.1183 \\
\hline RV & 0.0892 & 0.1081 & 0.1096 & -0.1176 & 0.1387 & -0.0126 & 0.1167 & $\mathbf{0 . 1 7 5 5}$ \\
\hline r value & $\mathbf{0 . 5 9 9 6}$ & $\mathbf{0 . 6 9 3 2}$ & $\mathbf{0 . 8 0 4 9}$ & $\mathbf{- 0 . 6 8 7 1}$ & $\mathbf{0 . 8 1 6}$ & $\mathbf{- 0 . 0 8 0 1}$ & $\mathbf{0 . 6 8}$ & $\mathbf{0 . 7 7 7 4}$ \\
\hline
\end{tabular}


Table.7b Estimates of direct and indirect effects of physiological parameters on yield at phenotypic level under control condition

\begin{tabular}{|c|c|c|c|c|c|c|c|c|}
\hline Traits & $\mathbf{X}_{1}$ & $\mathbf{X}_{2}$ & $\mathbf{X}_{\mathbf{3}}$ & $\mathbf{X}_{4}$ & $\mathbf{X}_{5}$ & $X_{6}$ & $\mathbf{X}_{7}$ & $\mathbf{X}_{8}$ \\
\hline $\mathbf{X}_{1}$ & 0.1610 & -0.0072 & 0.0102 & -0.0374 & 0.0043 & 0.0047 & 0.0064 & -0.0052 \\
\hline $\mathbf{X}_{2}$ & 0.0014 & -0.0308 & -0.0021 & -0.0059 & -0.0074 & -0.0050 & -0.0023 & -0.0086 \\
\hline $\mathbf{X}_{3}$ & -0.0059 & -0.0064 & $\mathbf{- 0 . 0 9 2 7}$ & 0.0352 & -0.0348 & -0.0034 & -0.0232 & -0.0344 \\
\hline $\mathbf{X}_{4}$ & 0.0123 & -0.0102 & 0.0201 & -0.0531 & 0.0007 & 0.0129 & -0.0058 & 0.0117 \\
\hline $\mathbf{X}_{5}$ & 0.0102 & 0.0925 & 0.1446 & -0.0049 & 0.3855 & -0.0476 & 0.2798 & 0.1339 \\
\hline $\mathbf{X}_{6}$ & -0.0119 & -0.0665 & -0.0148 & 0.0989 & 0.0504 & -0.4082 & 0.2908 & -0.0367 \\
\hline $\mathbf{X}_{7}$ & -0.0195 & -0.0367 & -0.1234 & -0.0534 & -0.3570 & 0.3505 & -0.4920 & -0.0871 \\
\hline $\mathbf{X}_{8}$ & -0.0028 & 0.0246 & 0.0326 & -0.0193 & 0.0306 & 0.0079 & 0.0156 & 0.0880 \\
\hline r value & 0.1448 & -0.0407 & -0.0255 & -0.0399 & 0.0723 & -0.0882 & 0.0693 & 0.0616 \\
\hline
\end{tabular}


Correlation and path analysis between yield and physiological traits

Even though the selection for yield is the main criteria under drought other secondary traits play an important role in drought tolerance selection for these traits indirectly contribute to yield under drought stress situations. Physiological parameters play an important role in drought tolerance under stress condition. Phenotypic correlation and path coefficient analysis between yield and physiological traits under drought and control condition are presented in Table 6 and 7 respectively.

Chlorophyll content, relative water content, proline content, root length, root to shoot ratio and root volume showed significant positive correlation with yield per plant under drought condition. Whereas leaf rolling under drought stress situation showed significant negative correlation. Manickavelu et al., (2006) also reported significant and positive correlation of yield with relative water content, root length and root to shoot ratio, but leaf rolling reported significant negative correlation with yield. Santosh et al., (2014) reported significant positive correlations with proline content and relative water content under drought stress environment.

Path analysis of physiological characters contributing to drought tolerance on yield under stress situation helps know the effect of these parameters on dependent character yield. In the present study chlorophyll content, root length and root volume exhibited positive direct effect on yield under both drought stress and control condition. Under drought condition relative water content, proline content showed positive direct effect. Similar results were reported by Manickavelu et al., (2006) they showed relative water content and root to shoot ratio showed positive direct effect on yield. Uday (2013) reported that root length and root number had positive direct effect on yield.

The present study was conducted to tackle the problem of climate change by identifying and providing diverse parents for drought tolerance breeding. Study showed the presence of variation between the genotypes for yield and physiological traits under both drought and control conditions. The genotypes Sannavalya, Manjakaime, Gangadace, Najarbad, Madras sannaand JGL1798 exhibited drought tolerance. These lines performed better under drought condition in terms of yield attributing traits like productive tillers per plant, number of spikelets per panicle, number of seeds per panicle, yield per plant and test weight. Highest chlorophyll content, root length, root volume, relative water content and lowest leaf rolling score was recorded in these genotypes. Hence, these lines showed better tolerance to drought.

\section{References}

ABD Allah, A. A., Ammar, M. H. and Badawi, A.T., 2010, Screening rice genotypes for drought resistance in Egypt. J. Plant Breed. Crop Sci., 2(7): 205-215.

Alok, K., Rangare, N. R. and Vidyapati, V., 2013, Study of genetic variability of Indian and Exotic rice germplasm in Allahabad agroclimate. The Bioscan., 8(4): 1445-1451.

Amelia, H., Andrew, J. C., Tristram, C. B., Rolando, O. T. and Rachid, S., 2012, Root attributes affecting water uptake of rice (Oryzasativa) under drought. $J$. Exp. Bot., 63(13): 4751-4763.

Bates, L. S., Waldren, R. P. and Teare, I. D., 1973, Rapid determination of free proline for water- stress studies. Plant and Soil.39: 205-207.

Beena, R., Thandapani, V. and Chandrababu, R., 2012, Physio-morphological and 
biochemical characterization of selected recombinant inbred lines of rice for drought resistance. Indian J. Plant Physiol., 17(2): 189-193.

Dwivedi, J. L., 2007, Role of roots in conferring drought tolerance in rice. Paper presented in: Int. Symp. Root Bio. And MAS Strategies for Drought Resistance Improvement in Rice, Univ. Agric. Sci., Bangalore. September 2629, pp.14-15.

Fangjun, F., Xiaoyan, X., Xinbing, D., Hanhua, T., Lijun Luo. and Hanwei, M., 2012, Assessment of drought resistance among wild rice accessions using a protocol based on single-tiller propagation and PVC-tube cultivation. Aust. J. Crop Sci., 6 (7):1204-1211.

Fisher, R. A. and Yates, F., 1936, Statistical tables for biological, agricultural and medical research. Oliver and Boyd, London, pp.46-63.

GANA, A. S., 2011, Screening and resistance of traditional and improved cultivars of rice to drought stress at Badeggi, Niger State, Nigeria. Agric. Biol. J. N. America., 2 (6): 1027-1031.

Ganapathy, S., Ganesh, S. K., Shanmugasundaram, P. and Chandra, B, R., 2010, Studies on root traits for drought tolerance in rice (Oryza sativa L.) under controlled (PVC pipes) condition. Electron. J. Plant Breed., 1(4): 1016-1020.

Islam, M. Z., Khalequzzaman, M., Bashar, M. K., Ivy, N. A., Haque, M. M. and Mian, A. K., 2016, Variability assessment of aromatic and fine rice germplasm in Bangladesh based on quantitative traits. Bio. Med. Res. Int., pp.14.

Jabasingh, C. and Saravana Babu.S., 2013, Proline content of Oryza sativa L. under water stress. J. Academia and Industrial Res., 2(7): 442-445.

Jibouri, 1958, Genetic variability and correlation studies in chillies. Prog.
Hort., 36: 113-117.

Johnson, H. W., Robinson, H. F. and Comstock, R. E., 1955, Estimates of genetic and environmental variability in soybean, Agron. J., 7(7): 314-318.

Kanbar, A., Shashidhar, H. E.and Hittalmani, S., 2002, Mapping of QTL associated with root and related traits in $\mathrm{DH}$ population of rice (Oryza sativa L.). Indian J. Genet., 62(4): 287-290.

Kanbar, A., Toorchi, M. and Shashidhar, H. E., 2009, Relationship between yield and root morphological characters in rainfed low land rice (Oryza sativa L.).Cereal Res. Communication, 37(2): 261-268.

Lenka, D. and Mishra, B., 1973, Path coefficient analysis of yield in rice varieties.Indian J. Agric. Sci., 43: 376379.

Luis, B., Gomez-Luciano., Ching, H. H. and Chih, W. W., 2012, Proline accumulation role on drought-induced rice genotypes. Conference paper, National Pingtung Univ. Sci and Tech., Taiwan.

Mahender. A., Dash, G. K., Swain. P. and Rao, G. J. N., 2014, Physiological and molecular profiling of rice genotypes under drought stress. Oryza, 51(1): 1224.

Maisura. Muhamad, A. C., Iskandar, L., AHMAD, J. AND HIROSHI, E., 2014, Some physiological character responses of rice under drought conditions in a paddy system. J. ISSAAS., 20(1): 104114.

Mana Kano-Nakata., Yoshiaki Inukai., LEN, J. W., Joel, D. L. C., Siopongco. and Akira Yamauchi., 2011, Root development, water uptake, and shoot dry matter production under water deficit conditions in two CSSLs of rice: functional roles of root plasticity. Plant Prod. Sci., 14(4): 307-317.

Manickavelu, A., Nadarajan, N., Ganesh. S, 
K., Ganamalar, R. P. and Chandra BABU, R., 2006, Drought tolerance in rice: Morphological and molecular genetic consideration. Plant Growth Regul., 50: 121-138.

Manjappa, Uday, G. and Shailaja, H., 2014, Identification of drought tolerant and high yielding $\mathrm{F}_{2}$ genotypes of rice under aerobic condition. Oryza., 51(4): 273278.

Santosh, K., Dwivedi, S. K., Singh, S. S., Jha, S. K., Lekshmy, S., Elanchezhian, R., Singh, O. N. and Bhatt, B.P., 2014, Identification of drought tolerant rice genotypes by analyzing drought tolerance indices and morpho physiological traits. SABRAO J. Breed. Gen., 46(2): 217-230.

Zulqamain, H., Abdul, R., Asrar, M., Saleemur, R., Aftab, I., Aamir, H., Usman, S., Muhammad, Y, N., Samta, Z., Zahid, MA. and Khalid, M., 2013, Comparison of associations among yield and yield components in rice (Oryza sativa L.) under simulated drought stress condition using multivariate statistics. Int. J. Sci. and Engg. Res., 4(8): 2229-5518.

\section{How to cite this article:}

Navya, G.T., B.M. Dushyanthakumar, R. Madhuri, K.N. Shubha and Gangaprasad, S. 2019. Studies on Morpho-Physiological Traits Associated with Drought Tolerance in Local Landraces of Rice (Oryza sativa L.). Int.J.Curr.Microbiol.App.Sci. 8(07): 1940-1951. doi: https://doi.org/10.20546/ijcmas.2019.807.231 